\title{
The complex enviroment around Cir $\mathrm{X}-1$
}

\author{
A. D'Aí, R. Iaria, T. Di Salvo, G. Lavagetto and N. R. Robba \\ Dipartimento di Scienze Fisiche ed Astronomiche, Università di Palermo, via Archirafi n.36, 90123 \\ Palermo, Italy.
}

\begin{abstract}
We present the results of a $54 \mathrm{ks}$ long Chandra observation of the peculiar source Cir X1. We perform a comparative analysis of X-ray spectra, selected at different flux levels of the source. This allows us to distinguish between a very hard state, at a low countrate, and a brighter, softer, highly absorbed spectrum during episodes of flaring activity. The spectrum of the hard state clearly shows emission lines of highly ionized elements. During the flaring state, the spectrum also shows strong resonant absorption lines, mostly in the Fe K region. Physical implications on the changing system geometry and plasma behavior is discussed and compared with past observations.
\end{abstract}

Keywords: accretion discs - stars: individual: Cir X-1 — stars: neutron stars - X-ray: stars $X$-ray: spectrum - X-ray: general

PACS: $97.80 . J p$

\section{INTRODUCTION}

Cir X-1 is a peculiar NS LMXB that shows ultra-relativistic radio resolved jets. The continuum X-ray emission consists of a thick Comptonized component, which, for relatively narrow band coverage, cannot be distinguished from a simpler sum of two thermal components, and a variable hard-tail above $10 \mathrm{keV} \mathrm{[1].} \mathrm{The} \mathrm{spectrum} \mathrm{shows}$ a phase-dependent evolution, whose major characteristic, when the source is near the periastron passage is the appearence of a large column density of neutral matter occulting the continuum X-ray emission. Its origin has been assigned or in the colder outer layers of the accretion disc, or alternatively in the occulting presence of the companion star [2]. In two past Chandra observations near the zero phase, the $X$-ray spectrum showed P-Cygni profiles of $\mathrm{H}$ - and He-like ions, whose intensity decreased with decreasing luminosity of the source [3]. It is argued that these features arise from a radiatively, or thermally-driven wind from a hard X-ray irradiated accretion disc.

\section{DATA ANALYSIS AND RESULTS}

Cir X-1 was observed with Chandra on 2005 June 02 using the HETGS, for a total integration time of $53 \mathrm{ks}$ (OBS. ID. 5478, from the Chandra public archive). In this work we use the 1st-order HEG and MEG spectra. Given the strong variability of the source we performed a time-selected spectral analy sis based on the flux variability of the source. In Fig.1, left panel, we show the source lightcurve. Arrows indicate the time selection adopted to extract the spectra. Spectral fitting is based on a continuum model with a single thermal component multiplied with a partial covering component $[2,3]$. Local features are fitted, adding locally Gaussians/edges to the continuum. In Xspec language:

\footnotetext{
CP968, Astrophysics of Compact Objects, International Conference on Astrophysics of Compact Objects edited by Y.-F. Yuan, X.-D. Li, and D. Lai

(C) 2008 American Institute of Physics 978-0-7354-0485-4/08/\$23.00
} 

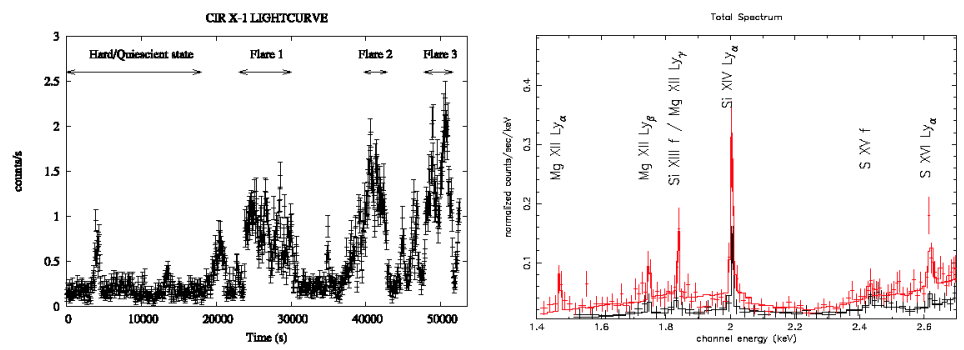

FIGURE 1. Left panel: Chandra observations of Cir X-1: lightcurve; right panel: emission line in the spectrum of $\mathrm{Cir} \mathrm{X}-1$ in the soft X-ray region
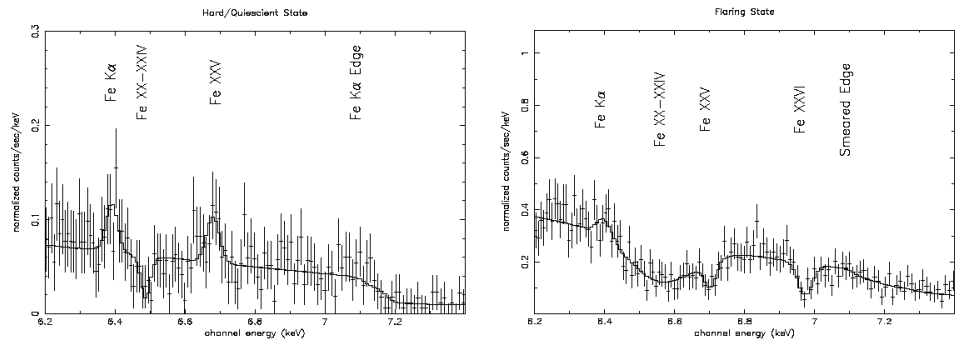

FIGURE 2. Left panel: the $6.27 .4 \mathrm{keV}$ region (QS); right panel: the $6.27 .4 \mathrm{keV}$ region (FS)

$w a b s^{*} p c f a b s^{*} e d g e^{*}(b b+$ gaussians $) . \mathrm{N}_{H}$ of the wabs component is fixed to $1.85 \times 10^{22}$ $\mathrm{cm}^{-3}$ [3]. We performed a time-selected spectral analysis for each state, marked with arrows in Fig. 1. We noted that spectra during the flare episodes show a strong similarity both in continuum and in the features. Therefore, we merge the flaring spectra into one spectrum, distinguishing only between the quiescienst state (QS) spectrum and the flaring state (FS) spectrum. During the QS the source is in a particular hard state. The fit requires only one component to fit the continuum, either a thermal blackbody of $\sim 3$ $\mathrm{keV}$ or rather flat power-law of photon-index $\sim 0$. Partial covering is required with a covering fraction of $70 \%$ and an associated column of $16 \times 10^{22} \mathrm{~cm}^{-3}$. During the flaring episodes the continuum strongly changes: it softens $(\sim 1.5 \mathrm{keV})$ and becomes more luminous (about two times the QS value). Partial covering now screens completely the radiative continuum (covering fraction close to unity), while the associated density column partially reduces $\left(\mathrm{N}_{H} \sim 9 \times 10^{22} \mathrm{~cm}^{-3}\right.$ ). The low energy spectrum shows, both during the QS and the FS emission lines of H-like (Mg XII, Si XIV, S XVI) and He-like elements (Ca XIX and Fe XXV). Moreover we also significantly detect the forbidden lines of Si XIII and S XV, but not the correspondent recombination and the resonant lines. From the line diagnostics of the He-like lines, and the ratio of the intensities of the $\mathrm{H}$-like and He-like ions we derived $[4,5]$ that the plasma is purely photo-ionized, it has a temperature of $10^{6}-10^{7} \mathrm{~K}$ and an electron density of $10^{13} \mathrm{~cm}^{-3}$. These features become more difficult to detect during the FS because of the higher photo-electric absorption 
and the appearence of strong absorption features. The mostly remarkable change in the features, in the passage from the QS to the FS, regards the iron 6.2-7.4 keV region. In the QS state (Fig.2, left panel) we observed the presence of the Fe $\mathrm{K} \alpha$ fluorescence line, a narrow absorption line at $6.5 \mathrm{keV}$, a broad Fe XXV emission line and a strong edge compatible with neutral iron. This landscape drastically changes during the FS (Fig. 2, right panel): the $\mathrm{Fe} \mathrm{K} \alpha$ is present but it becomes statistically hard to detect due to a broad absorption feature centered at $6.57 \mathrm{keV}$, with an equivalent width of $125 \mathrm{eV}$. The wing of this line embraces another absorption feature, compatible with $\mathrm{Fe}$ $\mathrm{XXV}$, while at higher energies we observe the strong blueshifted absorption line of Fe XXVI. At higher energies the spectrum falls rapidly off due to the presence of a smeared edge at $7.4 \mathrm{keV}$. We interpret the broad absorption feature as a blending of iron ions at intermediate ionization stages ( $\mathrm{Fe}$ XX - Fe XXIV), while the simultaneous presence of the $\mathrm{H}$ - and He- like ions indicate that the absorbing medium has a strong ionization gradient. Line broadening results from intense turbulent velocity fields that could be present in an optical thick outflow.

\section{CONCLUSIONS}

This new observation of Cir X-1 reveals new unknown aspects on this source. The richness and complexity of the features, their appearence and changing properties make Cir X-1 one of the best example where the physics of photo-ionized plasma in LMXB can be studied in detail. The main spectral continuum change beteween QS and FS is the partial covering fraction that strongly indicates a geometrically inflation of the occulting medium. The fact that this change is accompanied by the appearence of highly ionized absorption features leads to the conclusion that the rise in the column density of the occulting material goes from the innermost irradiated central zones out to the external border of the accretion disk. We interpret the spectral changing of the source mainly as due to a combination of geometrical factors and to a change in the irradiating flux: the source seems to undergo enhanced, but turbulent accretion, that inflates and thickens a corona, possibly present above the accretion disk. Optically thick accreting material present a blueshift of $370 \pm 100 \mathrm{~km} \mathrm{~s}^{-1}$ and a ionization factor $\log (\xi)$ between 3.5 and 4.2. Although this value is consistent with past observations, we note that the bulk motion velocity outflow is much less than the value reported by Schulz and Brandt [3], when the source showed the P-Cygni profiles. This might be due to a lower irradiating flux that is no longer able to consistently evaporate and accelarate the disk-fed wind.

\section{REFERENCES}

1. R. Iaria, L. Burderi, T. Di Salvo, A. La Barbera, and N. R. Robba, ApJ 547, 412-419 (2001), arXiv:astro-ph/0009183.

2. W. N. Brandt, A. C. Fabian, T. Dotani, F. Nagase, H. Inoue, T. Kotani, and Y. Segawa, MNRAS 283, 1071-1082 (1996), arXiv:astro-ph/9607018.

3. N. S. Schulz, and W. N. Brandt, ApJ 572, 971-983 (2002), arXiv: astro-ph/0112483.

4. D. Porquet, and J. Dubau, $A \& A S$ 143, 495-514 (2000), arXiv : astro-ph/ 0002319.

5. T. Kallman, and M. Bautista, ApJS 133, 221-253 (2001). 
Copyright of AIP Conference Proceedings is the property of American Institute of Physics and its content may not be copied or emailed to multiple sites or posted to a listserv without the copyright holder's express written permission. However, users may print, download, or email articles for individual use. 